\title{
A fourth-order one-step block method for solving a stiff ordinary differential equation
}

\begin{abstract}
In this paper, we propose an A-stable one-step block method of order four for solving a stiff ordinary differential equation. This method will approximate the solutions of a stiff ordinary differential equation at three points simultaneously using a constant step size. The method is similar to the one-step method and it is self-starting but the implementation is based on the predictor-and-corrector formulae. Several problems have been tested in this paper to prove the efficiency and accuracy of this method. Numerical results are presented to illustrate the performance of the proposed method. The results clearly show that the proposed method is able to produce comparable and better results compared to the existing methods when solving stiff differential equations.
\end{abstract}

Keyword: Newton's iteration; Stiff ordinary differential equation; Three-point block one-step method 